\title{
ARTICLE
}

Clinical Study

\section{Randomised phase II trial to investigate catumaxomab (anti-EpCAM $\times$ anti-CD3) for treatment of peritoneal carcinomatosis in patients with gastric cancer}

\author{
Maren Knödler ${ }^{1}$, Justus Körfer ${ }^{1}$, Volker Kunzmann ${ }^{2}$, Jörg Trojan ${ }^{3}$, Severin Daum ${ }^{4}$, Michael Schenk ${ }^{5}$, Frank Kullmann ${ }^{6}$, Sebastian Schroll ${ }^{7}$, \\ Dirk Behringer ${ }^{8}$, Michael Stahl ${ }^{9}$, Salah-Eddin Al-Batran ${ }^{10}$, Ulrich Hacker ${ }^{1}$, Stefan Ibach ${ }^{11}$, Horst Lindhofer ${ }^{12}$ and Florian Lordick $^{1}$
}

BACKGROUND: Peritoneal carcinomatosis (PC) represents an unfavourable prognostic factor for patients with gastric cancer (GC). Intraperitoneal treatment with the bispecific and trifunctional antibody catumaxomab (EpCAM, CD3), in addition to systemic chemotherapy, could improve elimination of PC.

METHODS: This prospective, randomised, phase II study investigated the efficacy of catumaxomab followed by chemotherapy (arm A, 5-fluorouracil, leucovorin, oxaliplatin, docetaxel, FLOT) or FLOT alone (arm B) in patients with GC and PC. Primary endpoint was the rate of macroscopic complete remission (mCR) of PC at the time of second diagnostic laparoscopy/laparotomy prior to optional surgery.

RESULTS: Median follow-up was 52 months. Out of 35 patients screened, 15 were allocated to arm A and 16 to arm B. mCR rate was $27 \%$ in arm $A$ and $19 \%$ in arm B $(p=0.69)$. Severe side effects associated with catumaxomab were nausea, infection, abdominal pain, and elevated liver enzymes. Median progression-free (6.7 vs. 5.4 months, $p=0.71)$ and overall survival ( 13.2 vs. 13.0 months, $p=0.97)$ were not significantly different in both treatment arms.

CONCLUSIONS: Addition of catumaxomab to systemic chemotherapy was feasible and tolerable in advanced GC. Although the primary endpoint could not be demonstrated, results are promising for future investigations integrating intraperitoneal immunotherapy into a multimodal treatment strategy.

British Journal of Cancer (2018) 119:296-302; https://doi.org/10.1038/s41416-018-0150-6

\section{INTRODUCTION}

Peritoneal carcinomatosis (PC) is a common manifestation of relapse or primary metastatic spread in patients with gastric cancer (GC). Survival outcomes are poor. ${ }^{1}$ Due to variable drug delivery to the peritoneum, it is still unclear, to which extent systemic chemotherapy is effective for PC. ${ }^{2}$ Nevertheless, systemic chemotherapy is considered the standard treatment for $\mathrm{PC}^{3}$ Effective strategies for treatment of PC are strongly needed. Besides to chemotherapy, local treatment approaches like cytoreductive surgery (CRS) plus hyperthermic intraperitoneal chemotherapy (HIPEC), which utilises surgery to reduce the visible tumour burden and HIPEC to eradicate peritoneal micrometastases are an option in treating PC in GC patients. Survival analyses after CRS plus HIPEC have shown that complete cytoreduction is associated with better overall survival (OS). ${ }^{4}$ However, a precise selection of patients (who should be in a good general condition, have a resectable primary gastric tumour and low peritoneal cancer burden) is highly recommended. ${ }^{5}$

Biologically targeted anti-cancer drugs in addition to systemic chemotherapy might be useful to treat PC. Catumaxomab (formerly marked by Fresenius Biotech, Munich, Germany) was developed and approved as a targeted therapy for the i.p. treatment of malignant ascites in cancers expressing the epithelial cell-adhesion molecule (EpCAM). ${ }^{6}$

EpCAM is physiologically expressed on epithelial tissues. In contrast, non-epithelial tissues like endothelium and mesenchymal tissues are EpCAM negative. Due to high-level expression on different epithelial tumours, EpCAM was considered as an interesting target for anticancer therapy. ${ }^{7}$ The cell surface protein is known to be overexpressed in $>90 \%$ of gastric tumours. ${ }^{7}$ In case of peritoneal application for PC, catumaxomab exclusively binds to epithelial tumour cells and not to the EpCAM-negative mesothelial

\footnotetext{
${ }^{1}$ University Cancer Center Leipzig (UCCL), University Hospital Leipzig, Leipzig, Germany; ${ }^{2}$ Department of Internal Medicine II, University Hospital Würzburg, Würzburg, Germany;

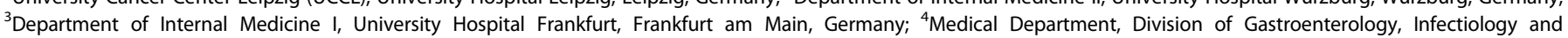

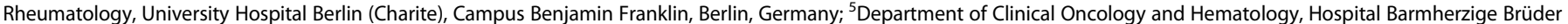

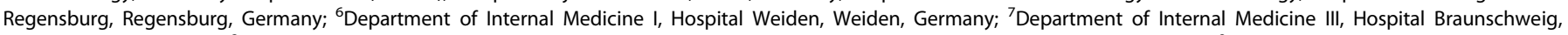

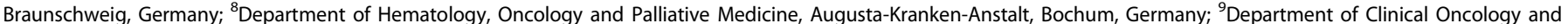

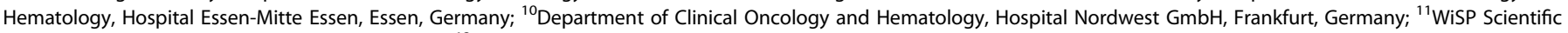
Service Pharma GmbH, Langenfeld, Germany and ${ }^{12}$ LINDIS Biotech GmbH, Planegg, Germany

Correspondence: Maren Knödler (maren.knoedler@medizin.uni-leipzig.de)

These authors contributed equally: Maren Knödler, Justus Körfer.
}

Received: 8 March 2018 Revised: 12 May 2018 Accepted: 21 May 2018

Published online: 10 July 2018 
cells of the peritoneal surface. Catumaxomab is a chimeric (ratmurine) bispecific and trifunctional antibody (trAb), which combines the characteristics of classical monoclonal antibodies and bispecific molecules. TrAbs have two antigen binding sites with two different specificities. Catumaxomab in particular binds three different cell types: (1) one antigen binding site does recognise $\operatorname{EpCAM}^{8}(2)$ the second antigen binding site connects to CD3 positive T cells ${ }^{9}$ and (3) the Fc region binds to type I, Ila, and III Fcy receptors $(F c \gamma R)$ on accessory cells of the immune system initiating the activation of accessory cells (e.g., macrophages, dendritic cells, and natural killer cells). ${ }^{10}$ Catumaxomab's antitumour effect is a result of a complex immune reaction at the tumour site involving $T$ cell-mediated lysis, antibody-dependent cell-mediated cytotoxicity and phagocytosis. ${ }^{8}$ Clinical trials have shown efficacy and acceptable tolerability of catumaxomab as i.p. treatment of malignant ascites in patients with ovarian and non- ovarian epithelial cancers. ${ }^{6,11}$ In a pivotal phase II/III study, patients with EpCAM-positive cancer (ovarian and non-ovarian cancer) presenting with symptomatic malignant ascites requiring therapeutic paracentesis were randomised to receive paracentesis plus i.p. catumaxomab or paracentesis alone. Catumaxomab led to a significantly longer puncture-free-survival time (a composite endpoint of time free of paracentesis and OS time) and prolonged time to progression. Subgroup analyses indicated that patients with advanced GC may benefit from this therapy with regard to OS, while other pre-planned analyses (ovarian cancer patients and non-ovarian cancer patients) did not reveal subgroup-specific OS improvements.

Most commonly reported adverse side effects of i.p. catumaxomab treatment were infusion-related abdominal pain and signs of the proposed immunological mechanism of action (e.g., pyrexia, nausea and vomiting). These events were

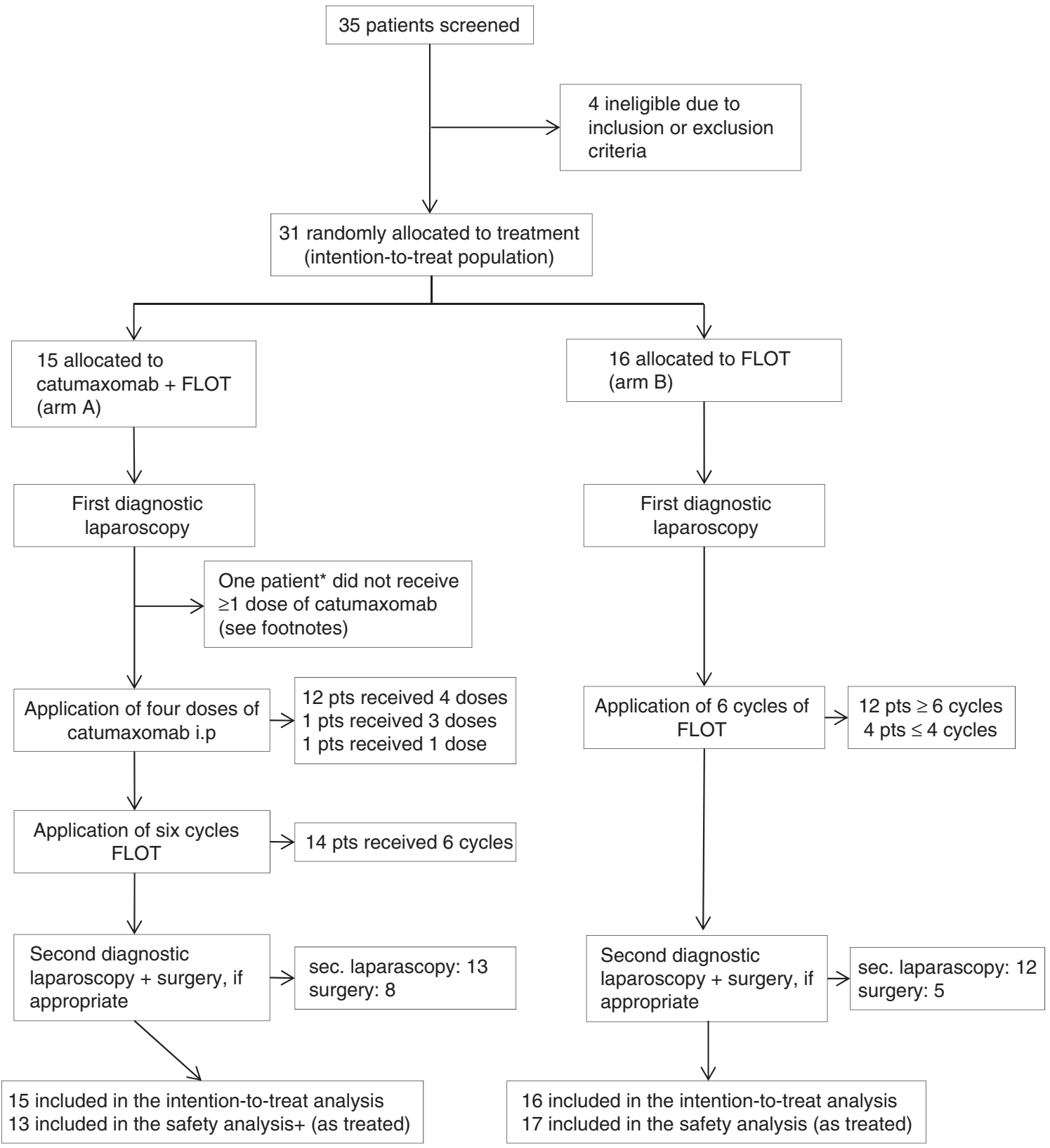

Fig. 1 Trial profile depicting the total number of patients at screening, inclusion, randomisation, and analysis. FLOT 5-fluorouracil, leucovorin, oxaliplatin and docetaxel, i.p. intraperitoneal, pts patients. *one patinet did not receive at least one dose of catumaxomab and was switched to the FLOT arm (for safety population only). +one patient died after three doses of catumaxomab and did not receive FLOT treatment 
generally mild-to-moderate in intensity and mostly fully reversible. ${ }^{6}$

In summary, PC in GC is a significant health problem resulting in poor prognosis and with no specific standard treatment. New and more effective therapies for prevention and treatment of PC are urgently needed. This phase II study evaluated the role of i.p. administration of catumaxomab followed by standard systemic chemotherapy as new multimodal treatment approach for patients with GC and macroscopic PC. The main objective was to investigate the efficacy of catumaxomab by determination of the rate of macroscopic complete remissions (mCR) of PC after treatment with one cycle (four doses) of catumaxomab followed by six cycles of chemotherapy with 5-fluorouracil, leucovorin, oxaliplatin and docetaxel (FLOT).

\section{PATIENTS AND METHODS}

Study design and patients

This multicentre prospective, randomised, open-label phase II study was sponsored by the Arbeitsgemeinschaft Internistische Onkologie (AIO) Studien $\mathrm{gGmbH}$, registered under accession number NCT01504256 at ClinicalTrials.gov and activated in 11 centres in Germany. The study aimed to investigate the efficacy and safety of systemic treatment with i.p. catumaxomab prior to FLOT chemotherapy or FLOT alone in patients with GC and PC at primary diagnosis. Patients aged $>18$ years with a histologically confirmed diagnosis of GC including oesophagogastric junction cancer (EGJ) type II and type III, according to Siewert's classification were eligible if they had a macroscopic PC (stages P1-4 according to Gilly's classification ${ }^{12}$ ), an Eastern Cooperative Oncology Group (ECOG) performance status of 0 or 1 , medically fit for potential gastrectomy after primary systemic (and i.p.) treatment, adequate organ functions, and a life expectancy of at least 12 weeks. Selection criteria excluded patients with distant metastasis other than PC, clinically significant cardiovascular disease $<1$ year before enrolment, history of HIV infection or chronic hepatitis B or C, active and clinically serious infection and pre-existing neuropathy >grade 1 .

Study procedures and endpoints

Diagnostic laparoscopy was performed to define a baseline status of the extent of PC prior to enrolment. All lesions were photographically documented during laparoscopy. Documentation of the greatest diameter of the lesions in each of the four quadrants was required. Classification of PC was done according to the Gilly PC index ${ }^{12}$ and scored according Sugarbaker's PC index $(\mathrm{PCl}){ }^{13}$ Patients were randomly assigned to one of two treatment arms in a 1:1 ratio. Patients allocated to arm $A$ received i.p. catumaxomab administered over $3 \mathrm{~h}$ via a constant infusion pump system at escalating doses of $10,20,50$, and $150 \mu \mathrm{g}$ on days $0,3,7$, and 10 . Intravenous paracetamol $1000 \mathrm{mg}$ was administered prior to catumaxomab for prophylaxis of cytokine-release associated symptoms. After 7 days the last infusion of catumaxomab, six cycles of FLOT chemotherapy (5-fluorouracil $(2600 \mathrm{mg} /$ $\mathrm{m}^{2}$ as $24 \mathrm{~h}$ infusion, $\left.\mathrm{d} 1\right)$, leucovorin $\left(200 \mathrm{mg} / \mathrm{m}^{2}, \mathrm{~d} 1\right)$, oxaliplatin $\left(85 \mathrm{mg} / \mathrm{m}^{2}, \mathrm{~d} 1\right)$, docetaxel $\left(50 \mathrm{mg} / \mathrm{m}^{2}, \mathrm{~d} 1\right)$, q2wk) were administered. In arm B patients received six cycles of FLOT without prior catumaxomab. After completing catumaxomab plus chemotherapy or chemotherapy alone, imaging-based response assessment was done and evaluated locally according to RECIST version 1.1 criteria $^{14}$ and a second diagnostic laparoscopy or laparotomy was recommended for evaluating disease response and assessing the possibility of resection. In case of complete resectability, resection of the primary tumour by gastrectomy and peritonectomy including all macroscopically involved parts of the peritoneum were recommended as a treatment option. Primary endpoint was the rate of macroscopic complete remission (mCR) of PC, defined as disappearance of all peritoneal lesions on $\mathrm{CT}$ and second
Table 1. Baseline demographics and clinical characteristics of the study population

\begin{tabular}{|c|c|c|}
\hline & $\begin{array}{l}\text { Catumaxomab + FLOT } \\
\text { (Arm A) } N=15\end{array}$ & $\begin{array}{l}\text { FLOT (Arm B) } \\
N=16\end{array}$ \\
\hline \multicolumn{3}{|l|}{ Age, years } \\
\hline $\begin{array}{l}\text { Median (range, } \\
\text { IQR) }\end{array}$ & $56(25-77,49-63)$ & $52(38-73,43-62)$ \\
\hline Sex, male & 7 (47\%) & $10(62 \%)$ \\
\hline \multicolumn{3}{|c|}{ ECOG performance status } \\
\hline 0 & 7 (50\%) & $8(50 \%)$ \\
\hline 1 & 7 (50\%) & 7 (44\%) \\
\hline 2 & - & $1(6 \%)$ \\
\hline \multicolumn{3}{|l|}{ Primary site } \\
\hline Stomach & $14(93 \%)$ & $11(69 \%)$ \\
\hline $\begin{array}{l}\text { Oesophago-gastric } \\
\text { junction }\end{array}$ & $1(7 \%)$ & $5(31 \%)$ \\
\hline \multicolumn{3}{|l|}{ TNM-Classification } \\
\hline T3 & $9(60 \%)$ & $10(63 \%)$ \\
\hline $\mathrm{T} 4$ & $4(27 \%)$ & $4(25 \%)$ \\
\hline Tx & $2(13 \%)$ & $2(12 \%)$ \\
\hline No & $3(20 \%)$ & $3(19 \%)$ \\
\hline $\mathrm{N}+$ & $10(67 \%)$ & $11(69 \%)$ \\
\hline $\mathrm{Nx}$ & $2(13 \%)$ & $2(12 \%)$ \\
\hline $\begin{array}{l}\text { M1 (Peritoneal } \\
\text { carcinomatosis) }\end{array}$ & $15(100 \%)$ & 16 (100\%) \\
\hline \multicolumn{3}{|l|}{ Gilly classification } \\
\hline P1 & $1(7 \%)$ & $1(6 \%)$ \\
\hline $\mathrm{P} 2+\mathrm{P} 3$ & $9(60 \%)$ & $10(63 \%)$ \\
\hline P4 & $5(33 \%)$ & $5(31 \%)$ \\
\hline \multicolumn{3}{|c|}{ Peritoneal Cancer Index (PCI) } \\
\hline $\begin{array}{l}\text { Median (range, } \\
\text { IQR) }\end{array}$ & $8(1-34,5-17)$ & $12(1-33,3-19)$ \\
\hline
\end{tabular}

laparoscopy (if done), based on the intention-to-treat approach. Secondary endpoints included surgical resection rate (R0, R1, R2), OS, disease-free survival, progression-free survival (PFS) as well as frequency and severity of adverse events. Follow-up every 12 weeks for up to 1 year was requested in order to assess survival-related secondary objectives. Adverse events were assessed according to the National Cancer Institute Common Terminology Criteria for Adverse Events (NCl-CTCAE) version 3.0.

\section{Statistical analysis}

The objective of this randomised phase II trial was to find evidence that the catumaxomab arm has a superior response rate compared with chemotherapy alone. Based on the findings from trials with systemic chemotherapy only, the $\mathrm{mCR}$ rate after standard treatment was expected to be not higher than $5 \%$. It was assumed, that this is similarly valid for PC. Thus, the experimental therapy arm including catumaxomab was to be rated as insufficiently active, if the true $\mathrm{mCR}$ rate was $5 \%$ or lower. The experimental therapy would be considered to be a highly promising candidate for further development, if the true $\mathrm{mCR}$ rate amounted to $20 \%$ or more. According to these parameters, and applying a standard two-stage phase II design by Simon, ${ }^{15}$ MiniMax option, $N=21$ eligible patients had to be randomised into the experimental arm to achieve a power of $80 \%$ with a type I error of 0.1. This incorporated an interim analysis on the first 12 patients allowing stopping for futility. A similar number of patients 
were to be randomised to the reference arm, to control for selection bias. All parameters were evaluated in an explorative or descriptive manner. Any $p$ values were considered to be descriptive, are two-sided and presented explicitly without referring to hypotheses or a significance level. $\mathrm{mCR}$ rate of PC was compared using Fisher's exact test. PFS and OS were estimated by the Kaplan-Meier method and compared using the logrank test.

\section{RESULTS}

Patient characteristics

Figure 1 shows the CONSORT diagram. Between October 2011, and December 2014, 35 patients were registered. When catumaxomab became no longer available in 2014 due to stop of production and marketing, the study protocol was amended accordingly and patient recruitment was stopped prematurely in December 2014. Four patients were excluded from analysis due to violation of selection criteria, as evaluated in a blinded pre-

\begin{tabular}{|lll|}
\hline Table 2. Response rate & \\
\hline $\begin{array}{l}\text { Macroscopic complete } \\
\text { remission of peritoneal } \\
\text { carcinomatosis }\end{array}$ & $\begin{array}{l}\text { Catumaxomab + FLOT } \\
(N=15)\end{array}$ & $\begin{array}{l}\text { FLOT } \\
(N=16)\end{array}$ \\
\hline Complete remission (CR) & $4(27 \%)$ & $3(19 \%)$ \\
Non CR & $9(60 \%)$ & $9(56 \%)$ \\
No data & $2(13 \%)$ & $4(25 \%)$ \\
Clinical response & Catumaxomab + FLOT & FLOT \\
(RECIST) & $(N=13)$ & $(N=13)$ \\
\hline Partial response & $6(46 \%)$ & $6(46 \%)$ \\
Stable disease & $3(23 \%)$ & $2(15 \%)$ \\
Progressive disease & $3(23 \%)$ & $5(38 \%)$ \\
Not evaluated & $1(8 \%)$ & - \\
\hline CR complete remission, RECIST response evaluation criteria in solid tumours \\
\hline
\end{tabular}

analysis, leaving 31 patients evaluable for final analysis. Of these, 15 were randomly allocated to catumaxomab plus FLOT (arm A) and 16 to FLOT only (arm B). Out of 15 patients allocated to arm A, 14 received at least one dose of catumaxomab. The full set of four applications of catumaxomab could be administered to 12 patients (86\%). In arm A, 14 patients (100\%) obtained six cycles of FLOT. In arm B, six cycles or more of systemic chemotherapy could be administered to 12 patients (75\%). Table 1 delineates demographics and baseline disease characteristics.

Efficacy

Four patients $(27 \%)$ in the catumaxomab arm (exact $95 \% \mathrm{Cl}$ : $0.08-0.55)$ and three patients (19\%) in the chemotherapy alone arm (exact 95\% Cl: 0.04-0.55) showed $\mathrm{mCR}$ after systemic treatment. This difference in favour of arm A was not statistically significant $(p=0.69)$. Second laparoscopy/laparotomy for response assessment was not carried out in three patients in arm A and five patients in arm B. Regarding the clinical response (according to RECIST criteria) to systemic treatment, a partial response rate of $46 \%$ was achieved in both arms (Table 2).

At time of analysis, median follow-up was 52 months. Ten patients in arm A (67\%) and 13 patients in arm B (81\%) had tumour progression. Median PFS was 6.7 months vs. 5.4 months in $\operatorname{arm~A}$ vs. $B(p=0.71)$ (Fig. 2). Median OS was 13.2 months in arm $A$ and 13.0 months in arm $B(p=0.97)$ (Fig. 3). At the time of evaluation, one patient in arm A (7\%) and one patient in arm B $(6 \%)$, respectively, were alive without tumour progression. Two patients (arm A: $N=2$ (13\%), arm B: $N=2 \quad(12 \%)$ ) in both treatment arms were still alive at the time of final analysis. Surgery of the primary tumour and peritonectomy were done in eight patients in arm A (53\%), and five patients in arm B (31\%). Reasons for no surgery were: early progression (28\%), no possibility of R0 resection in the peritoneum $(22 \%)$, or at other sites $(22 \%)$ and impaired performance status (11\%). Exploratory analysis showed that the $\mathrm{PCl}$ (dichotomised at the median) is a significant prognostic factor for PFS $(p=0.046)$. Patients who achieved a $\mathrm{mCR}$ in both arms were those with a PCl below the median.

Progression-free survival

All patients, by randomisation

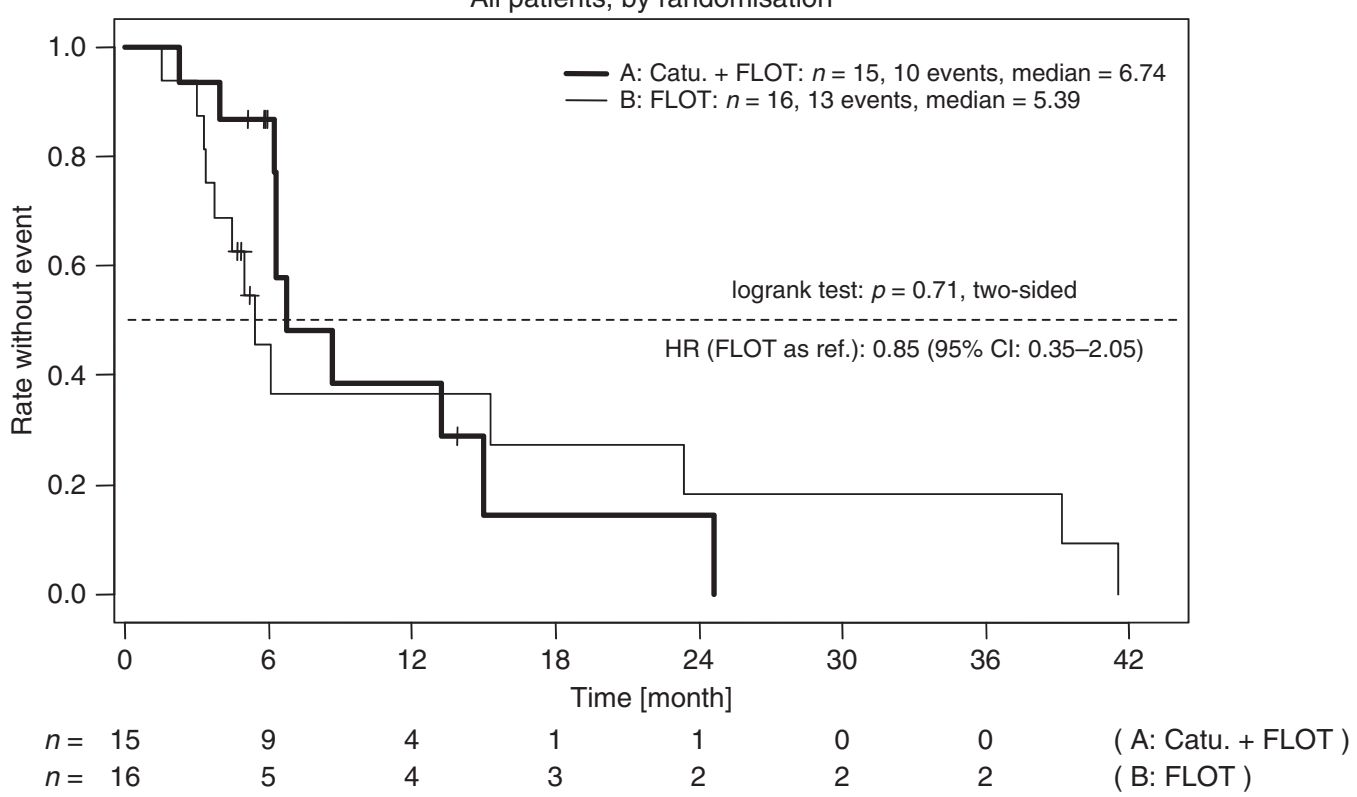

Fig. 2 Kaplan-Meier estimates for progression-free survival in the intention-to treat population. FLOT 5-fluorouracil, leucovorin, oxaliplatin and docetaxel, Catu Catumaxomab; HR hazard ratio, Cl confidence interval 
Overall survival

All patients, by randomisation

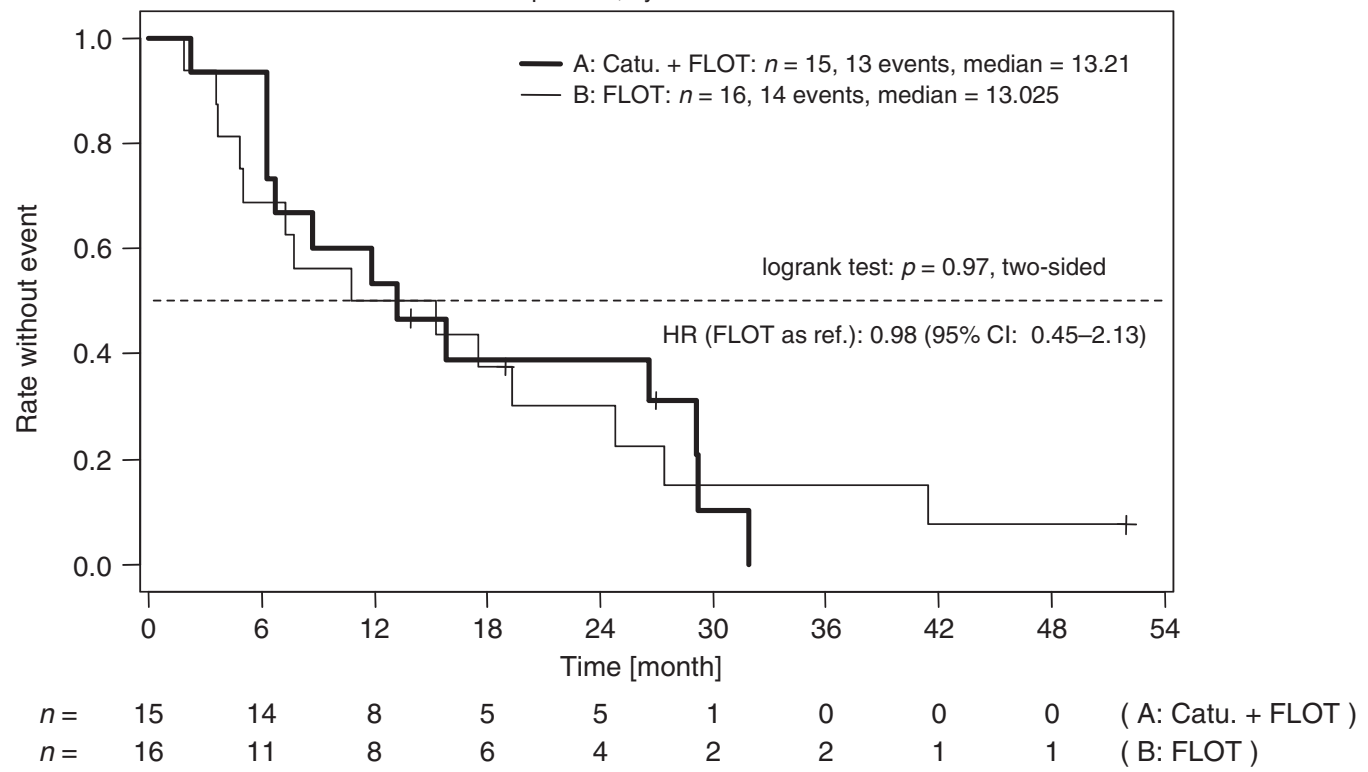

Fig. 3 Kaplan-Meier estimates for overall survival in the intention-to treat population. FLOT 5-fluorouracil, leucovorin, oxaliplatin and docetaxel; Catu: Catumaxomab; HR: hazard ratio; Cl: confidence interval

\begin{abstract}
Safety
Severe side effects (grade III/IV adverse events) associated with catumaxomab treatment prior to chemotherapy (arm A) were nausea (15\%), infection (23\%), abdominal pain (31\%), and elevated liver enzymes (gGT (31\%), bilirubin (23\%)). Four patients (29\%), experienced SAE's during catumaxomab treatment. Table 3 illustrates the reported adverse events in both arms that occurred during FLOT chemotherapy with a trend towards more adverse events following catumaxomab (arm A).
\end{abstract}

\section{DISCUSSION}

The aim of this study was to investigate the efficacy and safety of systemic treatment with i.p. catumaxomab in addition to systemic chemotherapy or chemotherapy alone in patients with GC and PC at primary diagnosis. Unfortunately, the primary endpoint of this study - improvement of the $\mathrm{mCR}$ rate of PC - was not met. A trend towards superiority of the experimental arm did not reach statistical significance ( $27 \%$ in arm A vs. $19 \%$ in arm B). However, the $\mathrm{mCR}$ rate in the standard arm $B$ was significantly higher compared to the assumptions made for sample size calculation. Nonetheless, i.p. treatment with catumaxomab in this disease setting showed an acceptable safety profile and addition of catumaxomab to six cycles of FLOT chemotherapy did not lead to any unexpected adverse events: the toxicity profile was roughly the same as in previous studies where catumaxomab was mostly investigated in patients with malignant ascites. ${ }^{16,17}$ However, we observed a trend towards more adverse events during FLOT chemotherapy following catumaxomab (arm A) compared with chemotherapy alone (arm B), without preventing the feasibility of systemic chemotherapy. The most frequent severe side effects associated with catumaxomab were nausea, infection, abdominal pain, and elevated liver enzymes.

Median PFS was 6.7 months in arm A vs. 5.4 months in arm B and median OS was 13.2 months and 13.0. So we have to conclude that no major differences in survival outcomes between the two arms were seen. Of note, both the efficacy and safety assessment are limited by small patient numbers. However, the survival outcomes are within the expected range for a palliative treatment approach in patients with metastatic GC, though median OS in both arms was longer than expected in a stage IV GC population with primary PC. ${ }^{18}$ One may argue that this could be due to the selection of patients with PC only, without other distant disease manifestations, and therefore, probably a generally lower tumour burden.

Although the primary endpoint was not met, the results are important and support the notion that future prospective trials investigating the application of i.p. therapy as part of a multimodal treatment strategy are feasible. Another important teaching of the actual study could be that the sequence immunotherapy followed by chemotherapy within 1 week is counterproductive. This holds true especially using $T$ cell redirecting bispecific antibodies, which are dependent on a functional immune system and where antitumour responses can last for weeks and even months. ${ }^{19,20}$ In the meantime, clinical investigations are available that the sequence, chemotherapy followed by immunotherapy, after a time period when the immune system has recovered from chemotherapy, is obviously better suited than vice versa. ${ }^{21,22}$ This fact should be taken into account for the design of upcoming studies using catumaxomab.

Other local treatment options in patients with $P C$ are under investigation. Recent studies comparing CRS with CRS plus HIPEC have shown a small but statistically significant survival improvement. ${ }^{23,24}$ However, CRS and HIPEC have also relevant morbidity and side effects. ${ }^{25}$ Therefore, a careful selection of patients who might benefit from this approach is important and is still to be defined. Data regarding the effectiveness and benefit of CRS and HIPEC in patients with GC und PC are still limited. Further clinical research on this approach is needed.

Our study has several limitations. The major limitation is the relatively small number of included patients which is in part a consequence of the premature closure of the trial due to the study drug catumaxomab becoming unavailable during the conduct of the study. Linked to this unforeseen problem, we observed a non-ideal balance between the PCl scores in both study arms which might have been more balanced with a higher number of recruited patients. In addition, a priori stratification of patients according to $\mathrm{PCl}$ at the timepoint of randomisation might have been useful. Another limitation is the lack of biological correlative analyses. It would be important to understand which 
Table 3. Adverse events in the safety population during FLOT chemotherapy

\begin{tabular}{|c|c|c|c|c|}
\hline & \multicolumn{2}{|c|}{$\begin{array}{l}\text { Catumaxomab + FLOT } \\
\text { (Arm A) }\end{array}$} & \multicolumn{2}{|c|}{ FLOT (Arm B) } \\
\hline & Any grade & $\begin{array}{l}\text { Grade } 3 \text { or } \\
4\end{array}$ & $\begin{array}{l}\text { Any } \\
\text { grade }\end{array}$ & $\begin{array}{l}\text { Grade } 3 \\
\text { or } 4\end{array}$ \\
\hline \multicolumn{5}{|l|}{ Adverse events } \\
\hline Nausea & $10(77 \%)$ & $1(8 \%)$ & $\begin{array}{l}12 \\
(71 \%)\end{array}$ & $3(18 \%)$ \\
\hline Vomiting & 5 (38\%) & $1(8 \%)$ & $7(41 \%)$ & $2(12 \%)$ \\
\hline Diarrhoea & 7 (44\%) & $0(0 \%)$ & $9(53 \%)$ & $1(6 \%)$ \\
\hline Fatigue & $5(38 \%)$ & $1(8 \%)$ & $\begin{array}{l}10 \\
(59 \%)\end{array}$ & $1(6 \%)$ \\
\hline Hand-foot syndrome & $4(31 \%)$ & $1(8 \%)$ & $4(24 \%)$ & $2(12 \%)$ \\
\hline Rash & $3(23 \%)$ & $0(0 \%)$ & $3(18 \%)$ & $0(0 \%)$ \\
\hline Anaemia & $13(100 \%)$ & $2(16 \%)$ & $\begin{array}{l}13 \\
(76 \%)\end{array}$ & $1(6 \%)$ \\
\hline Leukocytopaenia & $11(85 \%)$ & $1(8 \%)$ & $\begin{array}{l}14 \\
(82 \%)\end{array}$ & $1(6 \%)$ \\
\hline Neutropaenia & $12(92 \%)$ & $4(33 \%)$ & $\begin{array}{l}14 \\
(82 \%)\end{array}$ & $5(32 \%)$ \\
\hline Thrombocytopaenia & $5(38 \%)$ & $0(0 \%)$ & $3(18 \%)$ & $0(0 \%)$ \\
\hline Pyrexia & $4(30 \%)$ & $0(0 \%)$ & $4(24 \%)$ & $0(0 \%)$ \\
\hline Chills & 7 (54\%) & $2(15 \%)$ & $\begin{array}{l}11 \\
(65 \%)\end{array}$ & $1(6 \%)$ \\
\hline Febrile neutropaenia & $0(0 \%)$ & $0(0 \%)$ & $1(6 \%)$ & $1(6 \%)$ \\
\hline Infection & $3(23 \%)$ & $2(15 \%)$ & $4(24 \%)$ & $1(6 \%)$ \\
\hline Allergic reaction & $0(0 \%)$ & $0(0 \%)$ & $0(0 \%)$ & $0(0 \%)$ \\
\hline Mucositis/stomatitis & $1(8 \%)$ & $0(0 \%)$ & $6(35 \%)$ & $1(6 \%)$ \\
\hline Pain & $6(46 \%)$ & $0(0 \%)$ & $7(41 \%)$ & $1(6 \%)$ \\
\hline Dyspnoea & $2(15 \%)$ & $0(0 \%)$ & $1(6 \%)$ & $0(0 \%)$ \\
\hline Alopecia & 7 (54\%) & $0(0 \%)$ & $8(47 \%)$ & $0(0 \%)$ \\
\hline Elevated bilirubin & $1(8 \%)$ & $0(0 \%)$ & $0(0 \%)$ & $0(0 \%)$ \\
\hline \multicolumn{5}{|l|}{ Serious adverse events } \\
\hline Any & $3(23 \%)$ & & $5(29 \%)$ & \\
\hline None & $10(77 \%)$ & & $12(71 \%)$ & \\
\hline
\end{tabular}

subpopulation of patients benefits from i.p. catumaxumab. Nevertheless, we could demonstrate that a local treatment approach in patients with peritoneal metastatic GC seems to be feasible and should undergo further evaluation in clinical trials. Due to the low number of patients having isolated peritoneal metastases, conduction of clinical trials evaluating local treatment approaches in GC is challenging. However, we believe that for patients in a good performance status and with controlled disease during a defined time interval of systemic chemotherapy (e.g., 3-4 months) local therapy of peritoneal metastases deserves further evaluation.

In conclusion, the achievement of the primary endpoint of the study (improvement of the macroscopic CR rate of $P C$ ) unfortunately could not be demonstrated. In addition, no major differences in survival outcomes between the two arms were seen. However, i.p. immunotherapy in this disease setting showed an acceptable safety profile and addition of i.p. catumaxomab to systemic chemotherapy did not lead to any unexpected adverse events. The main achievement of this study, therefore was to demonstrate that the addition of an i.p. immunotherapy as part of a multimodal treatment approach was feasible in a multicentre setting and tolerable for patients with PC from GC.

\section{ACKNOWLEDGEMENTS}

We thank the patients and their families, investigators and colleagues at all participating centres.

\section{AUTHOR CONTRIBUTIONS}

F.L. was responsible for conception and study design; F.L., V.K., J.T., S.D., M.S., F.K., S.S., D.B., M.S., S.E.A.B., U.H. were involved in acquisition of data; M.K., J.K., F.L., S.I., were involved in analysis and interpretation of data; All authors contributed in writing, review, and/or revision of the manuscript.

\section{ADDITIONAL INFORMATION}

Competing interests: F.L. has received consulting fees from Astellas, BioNTech, Bristol-Myers-Squibb, Eli Lilly, Ganymed Pharmaceuticals, Merck Sharp \& Dohme; lecture honoraria from Amgen, Astra Zeneca, Eli Lilly, Merck Sharp \& Dohme, Servier and research support from Fresenius Biotech. V.K. has received research support from Fresenius Biotech. S.I. has received consulting fees from AIO $\mathrm{gGmbH}$. SEAB has an advisory role with Merck, Roche, Celgene, Lilly, and Nordic Pharma; S.I. is a speaker for Merck, Roche, Celgene, Lilly, and Nordic Pharma; and has received research grants from Sanofi, Merck, Roche, Celgene, Vifor, Medac, Hospira, and Lilly. H.L. is the CEO of Trion Research and inventor or coinventor of several trifunctional antibody patents. All other authors declare no competing interest.

Ethics approval and consent to participate: All patients provided written informed consent. Approvals for the study protocol and amendments were obtained from independent ethics committees. The study was conducted in Germany according to the Good Clinical Practice Guidelines and the latest version of the Declaration of Helsinki.

Consent for publication: This manuscript does not contain any individual person's data in any form.

Availability of data and material: The sponsor (AIO) was responsible for data management and statistical analysis and was supported by a contracted clinical research organisation (WiSP $\mathrm{GmbH}$, Langenfeld). The first authors and the senior author had full access to all study data and MK and FL had the final responsibility for the decision to submit for publication.

Funding: This work was supported by the Arbeitsgemeinschaft Internistische Onkologie $\mathrm{gGmbH}(\mathrm{AIO})$ as a legal sponsor of the trial (no grant numbers apply). The work was also supported by an unrestricted grant and drug supply (catumaxomab) by Fresenius Biotech (no grant numbers apply).

Note: This work is published under the standard license to publish agreement. After 12 months the work will become freely available and the license terms will switch to a Creative Commons Attribution 4.0 International (CC BY 4.0).

Publisher's note: Springer Nature remains neutral with regard to jurisdictional claims in published maps and institutional affiliations.

\section{REFERENCES}

1. D'Angelica, M. et al. Patterns of initial recurrence in completely resected gastric adenocarcinoma. Ann. Surg. 240, 808-816 (2004).

2. Jacquet, P. \& Sugarbaker, P. H. Peritoneal-plasma barrier. Cancer Treat. Res. 82, 53-63 (1996)

3. Smyth, E. C. et al. Gastric cancer: ESMO Clinical Practice Guidelines for diagnosis, treatment and follow-upt. Ann. Oncol. 27(Suppl. 5), v38-v49 (2016).

4. Sugarbaker, P. H. \& Yonemura, Y. Clinical pathway for the management of resectable gastric cancer with peritoneal seeding: best palliation with a ray of hope for cure. Oncology 58, 96-107 (2000).

5. Glehen, O. Cytoreductive surgery and intraperitoneal chemohyperthermia for peritoneal carcinomatosis arising from gastric cancer. Arch. Surg. 139, 20 (2004).

6. Heiss, M. M. et al. The trifunctional antibody catumaxomab for the treatment of malignant ascites due to epithelial cancer: results of a prospective randomized phase II/III trial. Int. J. Cancer 127, 2209-2221 (2010).

7. Went, P. et al. Frequent high-level expression of the immunotherapeutic target EpCAM in colon, stomach, prostate and lung cancers. Br. J. Cancer 94, 128-135 (2006).

8. Schmitt, M. et al. Opsonization with a trifunctional bispecific (alphaCD3 $\times$ alphaEp(AM) antibody results in efficient lysis in vitro and in vivo of EpCAM positive tumor cells by cytotoxic T lymphocytes. Int. J. Oncol. 25, 841-848 (2004). 
9. Zeidler, R. et al. Simultaneous activation of T cells and accessory cells by a new class of intact bispecific antibody results in efficient tumor cell killing. J. Immunol. Balt. 1950. 163, 1246-1252 (1999).

10. Zeidler, R. et al. The Fc-region of a new class of intact bispecific antibody mediates activation of accessory cells and NK cells and induces direct phagocytosis of tumour cells. Br. J. Cancer 83, 261-266 (2000).

11. Burges, A. et al. Effective relief of malignant ascites in patients with advanced ovarian cancer by a trifunctional anti-EpCAM $\times$ anti-CD3 antibody: a phase I/II study. Clin. Cancer Res. J. Am. Assoc. Cancer Res. 13, 3899-3905 (2007).

12. Gilly, F. N. et al. Regional chemotherapy (with mitomycin C) and intra-operative hyperthermia for digestive cancers with peritoneal carcinomatosis. HepatoGastroenterology 41, 124-129 (1994).

13. Sugarbaker, P. H. Management of peritoneal metastases - basic concepts. J. Balk. Union Oncol. 20, S2-S11 (2015). Suppl 1.

14. Eisenhauer, E. A. et al. New response evaluation criteria in solid tumours: revised RECIST guideline (version 1.1). Eur. J. Cancer 45, 228-247 (2009).

15. Simon, R. Optimal two-stage designs for phase II clinical trials. Control. Clin. Trials 10, 1-10 (1989).

16. Ströhlein, M. A. et al. Immunotherapy of peritoneal carcinomatosis with the antibody catumaxomab in colon, gastric, or pancreatic cancer: an open-label, multicenter, phase I/II trial. Onkologie 34, 101-108 (2011).

17. Bokemeyer, C. et al. A phase II study of catumaxomab administered intra- and postoperatively as part of a multimodal approach in primarily resectable gastric cancer. Gastric Cancer 18, 833-842 (2014).
18. Lordick, F., Lorenzen, S., Yamada, Y. \& Ilson, D. Optimal chemotherapy for advanced gastric cancer: is there a global consensus? Gastric Cancer 17, 213-225 (2014).

19. Bezan, A., Hohla, F., Meissnitzer, T. \& Greil, R. Systemic effect of catumaxomab in a patient with metastasized colorectal cancer: a case report. BMC Cancer 13, 618 (2013).

20. Petrelli, F., Borgonovo, K., Lonati, V., Elia, S. \& Barni, S. Regression of liver metastases after treatment with intraperitoneal catumaxomab for malignant ascites due to breast cancer. Target Oncol. 8, 291-294 (2013).

21. Heiss, M. M. et al. The role of relative lymphocyte count as a biomarker for the effect of catumaxomab on survival in malignant ascites patients: results from a phase II/III study. Clin. Cancer Res. 20, 3348-3357 (2014).

22. Romero, I. et al. Phase II trial of intraperitoneal (IP) administration of catumaxomab (C) as consolidation therapy for patients (pts) with relapsed epithelial ovarian cancer (OC) in second or third complete remission: GEICO 1001 study. J. Clin. Oncol. 32, 5528-5528 (2014). (15_suppl).

23. Yang, X.-J. et al. Cytoreductive surgery and hyperthermic intraperitoneal chemotherapy improves survival of patients with peritoneal carcinomatosis from gastric cancer: final results of a phase III randomized clinical trial. Ann. Surg. Oncol. 18, 1575-1581 (2011).

24. Bonnot, P. E. et al. CYTO-CHIP: cytoreductive surgery versus cytoreductive surgery and hyperthermic intraperitoneal chemotherapy for gastric cancer with peritoneal metastasis: a propensity-score analysis from BIG RENAPE and FREGAT working groups. J. Clin. Oncol. 36(4_suppl), 8-8 (2018).

25. Feingold, P. L., Kwong, M. L. M., Sabesan, A., Sorber, R. \& Rudloff, U. Cytoreductive surgery and hyperthermic intraperitoneal chemotherapy for gastric cancer and other less common disease histologies: is it time? J. Gastrointest. Oncol. 7, 87-98 (2016). 